\title{
In-situ Investigation of Multi-Step Lithiation of Tin Sulfide
}

Sooyeon Hwang ${ }^{1}$, Zhenpeng $\mathrm{Yao}^{2}$, Lei Zhang ${ }^{3}$, Maosen $\mathrm{Fu}^{1,4}$, $\mathrm{Kai} \mathrm{He}^{2}$, Liqiang Mai ${ }^{3}$, Chris Wolverton ${ }^{2}$ and Dong $\mathrm{Su}^{1}$

1. Center for Functional Nanomaterials, Brookhaven National Laboratory, Upton, United States

2. Department of Materials Science and Engineering, Northwestern University, Evanston, United States

3. State Key Laboratory of Advanced Technology for Materials Synthesis and Processing, Wuhan University of Technology, Wuhan, P. R. China

4. Shanxi Materials Analysis and Research Center, School of Materials Science and Engineering, Northwestern Polytechnical University, Xi'an, P. R. China

Materials adopting two-dimensional (2D) layered structure have been actively explored as electrode for lithium ion batteries since their unique crystal structures is beneficial for lithium ions to be intercalated between layers [1]. Metal chalcogenides which have 2D layered structure have demonstrated intriguing multi-step reaction with lithium ions; for example, it is known that lithiation of tin disulfide $\left(\mathrm{SnS}_{2}\right)$ takes place via intercalation, conversion, and alloying [2]. As electrochemical properties are highly dependent on how these complicated reactions proceed, investigation of the reaction pathways with in situ analysis is of importance to improve the electrochemical properties of electrode materials. However, thorough understanding of each reaction mechanism of $\mathrm{SnS}_{2}$ is still missing and full scenario of lithiation dynamics remains elusive.

In this work, we examine the dynamic lithiation process of tin disulfide using in situ transmission electron microscopy (TEM) and first-principles calculations [3]. Electron microscopy work was performed with a JEOL 2100F TEM at an accelerating voltage of $200 \mathrm{kV}$ with a Nanofactory STMTEM holder. Dry-format electrochemical cell setup was used for this work: $\mathrm{Li}_{1} / \mathrm{Li}_{2} \mathrm{O}$ on $\mathrm{W}$ tip as counter electrode/ solid electrolyte and $\mathrm{SnS}_{2}$ sample on $\mathrm{Cu}$ grid as active electrode and current collector. Changes in electron diffraction patterns are tracked during lithiation (Figure 1). Structural evolutions induced by lithium insertion are reflected in diffraction peak shift, appearance and disappearance of peaks; thus, we could distinguish reaction steps by the modifications in diffraction profiles. We find 4 sequential steps of lithiation reaction: intercalation, disordering, conversion and alloying, which is different from well-known three stages. Disordering step is suggested for the first time. After Li ions are intercalated between S-S layer, rock-salt phase is formed by the disordering of $\mathrm{Sn}$ and $\mathrm{Li}$ cations. As all the octahedral sites are filled with cations in rock-salt phase, intercalation channel can be restricted. In order for further lithiation, decomposition of rock-salt phase may be inevitable, resulting in a conversion reaction. First principles calculations are conducted not only to elucidate the ground state reaction pathways but to validate the founding from experiments. Due to discrepancies between lithiation reactions at equilibrium state and empirical results, we simulate non-equilibrium reaction pathways using non-equilibrium phase searching method [4]. Calculation results corroborate that rearrangement of cations would not increase the energy of whole system and the formation of rock-salt phase is energetically more favorable than other $\mathrm{LiSnS}_{2}$ polymorphs (Figure 2), which is well-matched with realtime TEM observation.

A combination of real-time analysis and DFT calculation here reveals the formation of intermediate phase during lithiation of $\mathrm{SnS}_{2}$, which cannot be achieved by post-mortem works and sheds light on the understanding of new reaction mechanisms of 2D electrode materials [5]. 
References:

[1] J.-M. Tarascon, M. Armand, Nature 414 (2001), P. 359.

[2] T.-J. Kim et al. J. Power Sources 167 (2007) p. 529.

[3] S. Hwang et al. submitted.

[4] Z. Yao et al. Chem. Mater. 29 (2017) p. 9011.

[5] This work is supported by the Center for Functional Nanomaterials, Brookhaven National Laboratory, which is supported by the U.S. Department of Energy (DOE), Office of Basic Energy Science, under Contract No. DE- SC0012704.
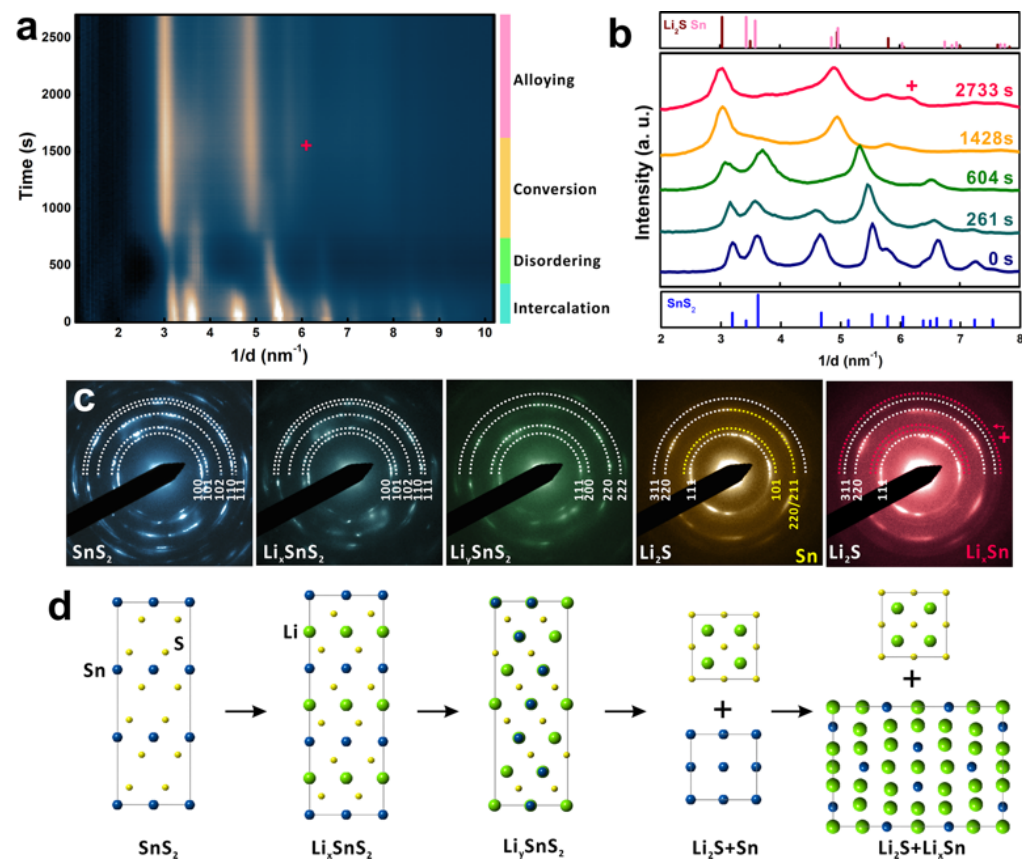

Figure 1. In situ selected area electron diffraction patterns of $\mathrm{SnS}_{2}$ during lithiation. (a) Electron diffraction intensity profile as a function of reaction time during in situ lithiation of $\mathrm{SnS}_{2}$. + denotes the evolution of Li-Sn alloy phase. (b) Radial intensity profiles of diffraction patterns at certain time. (c) Diffraction patterns corresponds to intensity profiles at (b). (d) Atomic models representing phase evolution during lithiation.

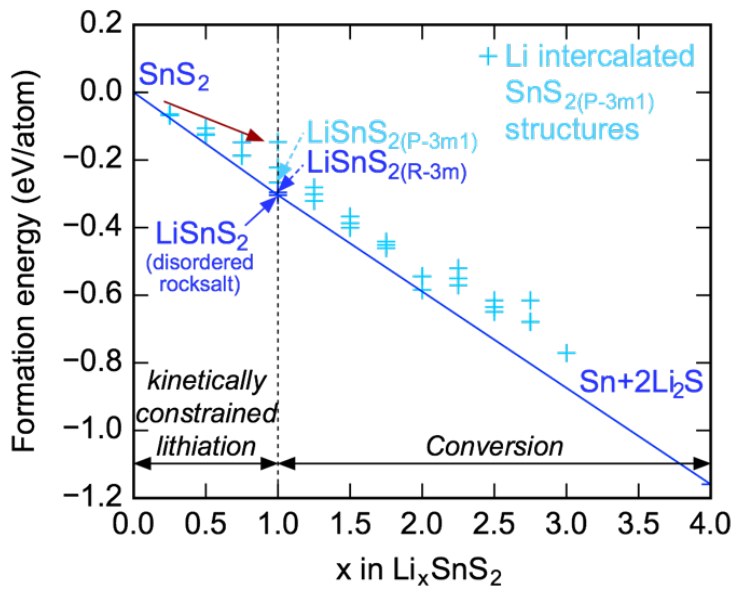

Figure 2. Convex hull generated with the calculated non-equilibrium phases of $\mathrm{Li}^{-\mathrm{SnS}} \mathrm{S}_{2}$. 